\title{
Chip-to-Chip Plasmonic Interconnects and the Activities of EU Project NAVOLCHI
}

\author{
A. Melikyan ${ }^{1 *}$, M. Sommer ${ }^{2}$, A. Muslija ${ }^{2}$, M. Kohl ${ }^{2}$, S. Muehlbrandt ${ }^{1,2}$, A. Mishra ${ }^{1}$, V. Calzadilla ${ }^{3}$, \\ Y. Justo ${ }^{4}$, J. P. Martínez-Pastor ${ }^{6}$, I. Tomkos ${ }^{7}$, A. Scandurra ${ }^{8}$, D. Van Thourhout ${ }^{5}$, Z. Hens ${ }^{4}$, M. Smit ${ }^{3}$, \\ W. Freude ${ }^{1,2}$, C. $\operatorname{Koos}^{1,2}$, J. Leuthold ${ }^{1,2}$ \\ 'Institute of Photonics and Quantum Electronics Karlsruhe Institute of Technology, 76131 Karlsruhe, Germany' \\ 2Institute of Microstructure Technology, Karlsruhe Institute of Technology, 76344 Karlsruhe, Germany \\ ${ }^{3}$ Dept. El. Engineering, Technische Universiteit Eindhoven, Postbus 513, 5600 MB Eindhoven, The Netherlands \\ ${ }^{+}$Physics and Chemistry of Nanostructure and ${ }^{5}$ Photonics Research Group, Ghent University, B-9000 Gent, Belgium \\ ${ }^{6}$ Instituto de Ciencia de los Materiales, Universidad de Valencia, 46980 Paterna, Spain \\ ${ }^{7}$ Athens Information Technology, PO Box 68, 19002 Athens, Greece \\ ${ }^{8}$ Interconnect Systems Group, STMicroelectronics srl, 95121 Catania, Italy \\ "Tel: (+49721)60842496,e-mail: argishti.melikyan@kit.edu
}

\section{ABSTRACT}

In this paper, the chip-to-chip interconnection architecture adopted by the EU-project NAVOLCHI are discussed. The plasmonic physical layer consisting of a plasmonic nanoscale laser, a modulator, an amplifier and a detector is introduced. Current statuses of the plasmonic devices are reviewed.

Keywords: plasmonic interconnects, Si plasmonic transceiver, NAVOLCHI.

\section{INTRODUCTION}

High speed chip-to-chip interconnects are essential for the realization of latest high-speed computing devices and ultra-compact system-in-a package solutions [1]. They are needed to further leverage products such as computers, mobile phones, HDTV, cameras etc. [2]. High speed I/Os with lowest power consumption can be realized by means of light and optical interconnects, which so far has shown to be capable of encoding up to $26 \mathrm{Tbit} / \mathrm{s}$ of data onto a single laser [3]. However, chip-to-chip optical interconnection requires high speed optical transceivers monolithically integrated on electronic ICs, which can convert digital information from electrical domain to optical domain and vice versa. Such transceivers should be compact, offer large bandwidth and consume little power in order to be economically and technologically efficient.

The most promising technology for realization of optical transceivers is the CMOS compatible silicon photonics platform whose low cost and large scale fabrication is ensured by well-developed advanced CMOS fabrication lines. Unfortunately, there is a size mismatch between silicon electronics and silicon photonics. Even state-of-the-art Si photonic transceivers are bulky comparing to electronic transistors [4]. Therefore, the integrated $\mathrm{Si}$ photonic transceivers would occupy large portion of the optoelectronic ICs making them economically not efficient, because of the expensive cost associated to every square micrometre in the CMOS fab.

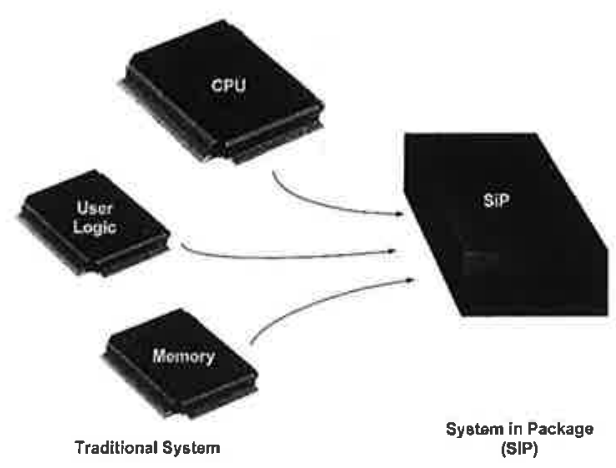

Figure 1. System in a Package (SiP) comprising a CPU, user logic and memory.

More recently, the field of plasmonics which deals with the manipulation of surface plasmon polaritons (SPP) has drawn the attention of the telecommunication community because of the possibility for ultra-compact dimensions by means of plasmonic devices [5-6]. The large bandwidths of plasmonic waveguides have also been demonstrated by the transmission experiments of $320 \mathrm{Gbit} / \mathrm{s}$ signal through SPP waveguides [7]. Various optically pumped SPP lasers have been demonstrated by researchers [8-12]. Moreover, M. Hill et al. have realized the smallest electrically pumped laser by making use of a plasmonic nanocavity [13]. Several plasmonic modulators have been proposed in the literature, with the footprints in a range of few micrometres [14-18]. While active 
plasmonic devices can still be useful because of their high speed and ultra-compact characteristics, passive plasmonic waveguides are less practical because of the high losses.

Here, the activities of EU project NAVOLCHI towards realization of compact Si-plasmonic transvers are discussed. The novel Si-plasmonic platform adopted by NAVOLCHI can be a solution for chip-to-chip interconnects where low loss silicon passive components are combined with high speed and ultra-compact plasmonic active devices such as light sources [8-13], modulators [14-18], amplifiers [19] and detectors [20-21]. In the following the current status of each plasmonic component comprising the NAVOLCHI's Si-plasmonic transceiver is reviewed.

\section{PLASMONIC DEVICE SPECIFICIATIONS}

The key plasmonic components studied by NAVOLCHI are the plasmonic nanolaser and the modulator which make the plasmonic transmitter. Further activities focus on the realization of plasmonic amplifiers and photodetectors which are the key components of a receiver. In the Si-plasmonic interconnection architecture light generated in plasmonic nanolaser is coupled into the $\mathrm{Si}$ nanowire waveguide which feeds the rest of the circuit with a continuous wave signal. After being modulated in the modulator, the light is then coupled out from the chip using a grating coupler. The optical signal is then transferred to the neighbouring chip through a passive optical link. The signal is detected by the plasmonic photodetector of the transceiver located on the second chip.

\subsection{Plasmonic Laser}

Electrically driven plasmonic nanolasers which are of importance for interconnect applications, have been realized by $\mathrm{M}$. Hill and co-workers [13]. These electrically pumped devices are based on InP/InGaAs/InP pillars with a conventional double heterostructures, see Fig. 2(a). The middle InGaAs active region with a height of $340 \mathrm{~nm}$ and a width of $130 \mathrm{~nm}$ is vertically and horizontally sandwiched between two InP and SiN layers respectively. The entire structure is then encapsulated in silver. The confinement along the longitudinal direction is achieved by fixing the length of the pillar to be $l$ and by the silver cladding which then provides the feedback. In Fig. 2(c) we give an example of the output power of the laser mode of a $3 \mu \mathrm{m}$ long device versus the pump current at $78 \mathrm{~K}$ temperature (red crosses). In addition, the spectrally integrated luminescence is given in Fig. 2(c) (blue circles). The plots clearly illustrate lasing above the threshold current as well as the carrier density pinning within the device.
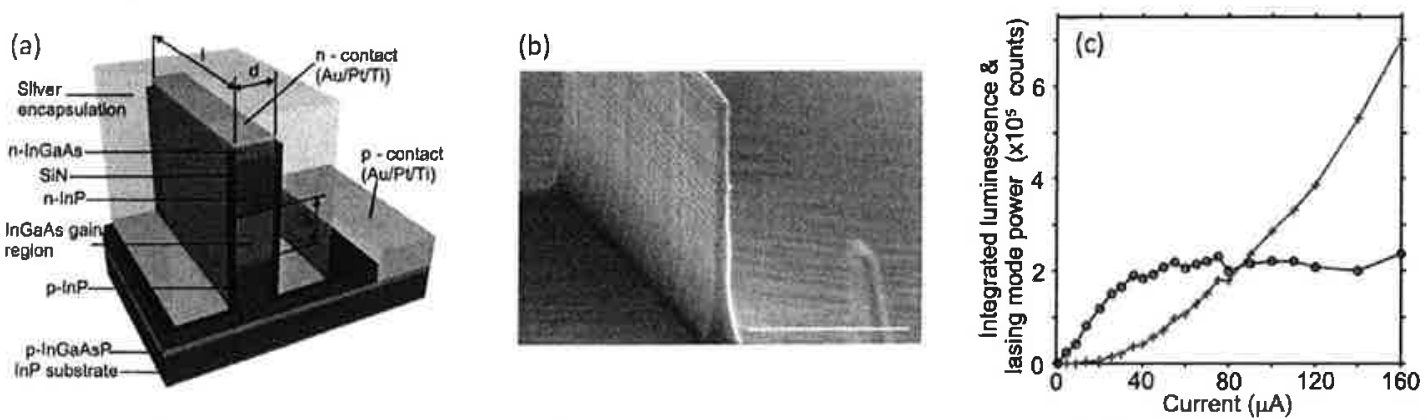

Figure 2. Plasmonic nanolaser and its configuration: (a) Schematic of the plasmonic heterostructure laser and (b) the scanning electron microscope picture of active part of the laser.(c) Lasing mode light output (red crosses), integrated luminescence (blue circles) for the device with $d=130 \mathrm{~nm}( \pm 20 \mathrm{~nm})$, versus current for $78 \mathrm{~K}$.

\subsection{Surface Plasmon Polariton Absorption Modulator}

A SPP absorption modulator (SPPAM) of ultra-compact size operating in the telecommunications window has recently been proposed [17]. The concept takes advantage of the strong plasmonic field confinement in an indium tin oxide (ITO) layer to modulate the absorption spectrum of SPPs via modulating the free carrier density of ITO. Absorption spectra of SPP for $\mathrm{Ag} / \mathrm{ITO}(8 \mathrm{~nm}) / \mathrm{Si}_{3} \mathrm{~N}_{4}(70 \mathrm{~nm}) / \mathrm{Ag}$ structure for two different ITO free carrier densities are depicted in Fig. 3(a). The absorption spectrum plotted in red is for the case when the free carrier density of ITO is increased by $5 \%$ relative to the free carrier density in the voltage off state. It can be seen that a small modulation of the free carrier density of ITO can lead to significant modulation of the SPP intensity. A first experimental proof of the modulation capability of such plasmonic absorption modulator has been given in [17] and is shown in Fig. 3(c-d). The measured extinction ratios are small because of the $60 \mu \mathrm{m}$ distance between the top and bottom electrodes, see Fig. 3(b). The concept has more recently been implemented with a much narrower insulator layer which resulted in an enhanced accumulation layer in ITO [18]. This way modulation extinction ratio of up to $20 \mathrm{~dB}$ have been demonstrated. 

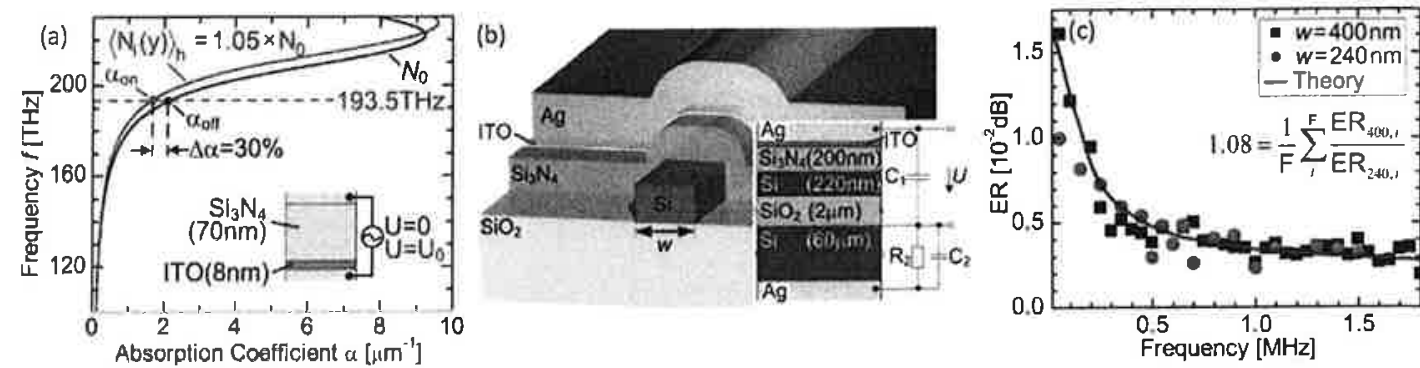

Figure 3. The dispersion relation (DR) of SPP at $\mathrm{Ag} / \mathrm{ITO} / \mathrm{Si}_{3} \mathrm{~N}_{1} / \mathrm{Ag}$ structure and the schematic of the device fabricated for proof-of-principle experiments: (a) DR of SPP at $\mathrm{Ag} / I T O(8 \mathrm{~nm}) / \mathrm{Si}_{3} \mathrm{~N}_{4}(70 \mathrm{~nm}) / \mathrm{Ag}$ for $10 V_{p p}$ voltage on and off states, (b) The schematic of the fabricated devices and (c) the measured extinction ratio.

\subsection{PLASMONIC AMPLIFIFER}

In order to overcome the plasmonic losses, a material system with a large coefficient of gain is necessary [19]. Here, we propose to use colloidal quantum dots for achieving compensation of plasmonic losses. Recently, $\mathrm{PbS}$ quantum dots embedded in $\mathrm{CdS}$ rods has been synthesized [22]. Optically pumping the rods enhanced photoluminescence (PL) has been reported with PL quantum yield of $45 \%-55 \%$ at the near-IR wavelength of $1200 \mathrm{~nm}$.

\section{CONCLUSIONS}

We introduced a novel interconnection architecture based on a Si-plasmonic platform. The physical layer of the interconnection is discussed in detail and the current status of each individual plasmonic device is given.

\section{ACKNOWLEDGEMENTS}

We acknowledge support by the EU FP7 project NAVOLCHI.

\section{REFERENCES}

[1] I. A. Young et al.: Optical I/O technology for tera-scale computing, IEEE J. of Solid State Circuits, vol. 45. pp. 235-248, Jan. 2010

[2] System-in-Package: The new wave in 3D packaging, TechSearch International, Inc., Sept. 2005

[3] D. Hillerkuss et al:: 26 Tbit s$^{-1}$ line-rate super-channel transmission utilizing all-optical fast Fourier transform processing, Nat. Photonics, vol. 5, pp. 364-371, May 2011.

[4] http://www intel.com

[5] M. Brongersma: Plasmonics for optical interconnection, in Proc, OFC, paper OTu2D.5, Mar. 2012.

[6] A. V. Zayats: Active plasmonics for optical interconnects, in Proc. OFC, paper OW3E. 1, Mar. 2012.

[7] S. Papaioannou et al: : $320 \mathrm{~Gb} / \mathrm{s}$-throughput capable $2 \times 2$ silicon-plasmonic router architecture for optical interconnects, J. Lightwave Technol., vol. 29, pp. 3185-3195, Nov. 2011.

[8] M. P. Nezhad et al.: Room-temperature subwavelength metallo-dielectric lasers, Nat. Photonics, vol. 4, pp. 395-399, Apr. 2010.

[9] R. Perahia et al.: Surface-plasmon mode hybridization in subwavelength microdisk lasers, Appl. Phys. Lett., vol. 95, pp. 201114, Nov. 2009.

[10] R.F. Oulton et al.: Plasmon lasers at deep subwavelength scale, Nature, vol. 461, pp. 629-632, Aug. 2009

[11] Ren-Min Ma et al: : Room-temperature sub-diffraction-limited plasmon laser by total internal reflection, Nat. Materials, vol. 10, pp. 110-113, Dec. 2010.

[12] M. A. Noginov et al: : Demonstration of a spaser-based nanolaser, Nature, vol. 460, pp. 1110-1112, Aug 2009.

[13] M. T. Hill et al.: Lasing in metallic-coated nanocavities, Nat. Photonics, vol. 1, pp. 589-594, Oct. 2007.

[14] J. A. Dionne et al: : PlasMOStor: A metal-oxide-Si field effect plasmonic modulator, Nano Lett., vol. 9 , pp. 897-902, Jan. 2009.

[15] E. Feigenbaum et al: : Unity-order index change in transparent conducting oxides at visible frequencies, Nano Lett., vol. 10. pp. 2111-2116, June 2010.

[16] W. Cai et al.: Compact, high-speed and power-efficient electrooptic plasmonic modulators, Nano Lett., vol. 9, pp. 4403-4411, Oct. 2009.

[17] A. Melikyan et al: Surface plasmon polariton absorption modulator, Opt. Express, vol. 19, pp. 8855-8869, April 2011.

[18] V. J. Sorger et al: $\lambda$-size silicon modulator, in Proc. CLEO, post-deadline paper CTh5D, May 2012

[19] P. Berini et al:: Surface plasmon-polariton amplifiers and lasers, Nat. Photonics, vol. 6, pp. 16-24, Dec. 2011.

[20] L. Goykhman et al, : Locally oxidized silicon surface-plasmon Schottky detector for telecom regime, Nano Lett., vol. 11, pp. 2219-2224, May 2011.

[21] M. W. Knight et al.: Photodetection with active optical antennas, Science, vol. 332, pp. 702-704, May 2011.

[22] Y. Justo et al.: Multiple dot-in-rod PbS/CdS heterostructures with high photoluminescence quantum yield in the nearinfrared, J. Am. Chem. Soc., vol. 134, pp. 5484-5487, Mar. 2012. 